Revue

Revue de l'histoire des religions

del'histoire des religions

Hervé MARTIN, Pérégrin d'Opole (vers 1260 - vers 1330). Un prédicateur dominicain à l'apogée de la chrétienté médiévale

Rennes, Presses Universitaires de Rennes, 2008, 218 p.-XVI p. de pl., $24 \mathrm{~cm}$ (« Histoire »), $16 €$.

Anne-Laure Lallouette

\title{
OpenEdition
}

Journals

Édition électronique

URL : http://journals.openedition.org/rhr/7733

DOI : $10.4000 /$ rhr.7733

ISSN : 2105-2573

Éditeur

Armand Colin

Édition imprimée

Date de publication : 1 mars 2011

Pagination : 116-120

ISBN : 978-2200-92685-4

ISSN : 0035-1423

Référence électronique

Anne-Laure Lallouette, «Hervé MARTIN, Pérégrin d'Opole (vers 1260 - vers 1330). Un prédicateur dominicain à l'apogée de la chrétienté médiévale », Revue de l'histoire des religions [En ligne], 1 | 2011, mis en ligne le 30 mai 2011, consulté le 17 octobre 2020. URL : http://journals.openedition.org/rhr/7733 ; DOI : https://doi.org/10.4000/rhr.7733

Ce document a été généré automatiquement le 17 octobre 2020

Tous droits réservés 


\section{Hervé MARTIN, Pérégrin d'Opole (vers 1260 - vers 1330). Un prédicateur dominicain à l'apogée de la chrétienté médiévale}

Rennes, Presses Universitaires de Rennes, 2008, 218 p.-XVI p. de pl., $24 \mathrm{~cm}$ (« Histoire »), $16 €$.

\section{Anne-Laure Lallouette}

\section{RÉFÉRENCE}

Hervé MARTIN, Pérégrin d'Opole (vers 1260 - vers 1330). Un prédicateur dominicain à l'apogée de la chrétienté médiévale, Rennes, Presses Universitaires de Rennes, 2008, 218 p.-XVI p. de pl., $24 \mathrm{~cm}$ (« Histoire »), $16 €$.

Inscrit dans la continuité des recherches menées par Hervé Martin à propos de la prédication, de la religion et des mentalités de la fin du Moyen Âge, cet ouvrage permet de découvrir précisément la personnalité et les travaux d'un prédicateur dominicain polonais qui vécut à la charnière des $\mathrm{XIII}^{\mathrm{e}}$ et $\mathrm{XIV}^{\mathrm{e}}$ siècles: Pérégrin d'Opole, et d'entrevoir à travers lui des aspects de la spiritualité d'une Europe orientale, souvent méconnue. La parution d'une édition critique du recueil homilétique de ce fervent propagateur de la foi a été l'occasion pour Hervé Martin de l'introduire auprès du public français, en faisant de son propre livre un outil qui permet de saisir le contexte de rédaction de ce manuel, d'en souligner les singularités mais aussi la profonde conformité par rapport aux modèles dont il s'inspire, et enfin d'en éclairer les traits les plus marquants. La figure de Pérégrin d'Opole est d'autant plus précieuse qu'elle sert de biais par lequel l'auteur entraîne son lecteur à la découverte du paysage ecclésiastique et politique de la Silésie, la Pologne occidentale et la Bohême médiévales, que des cartes permettent de situer précisément; et qu'elle aide, par un effet de métonymie, à saisir 
les évolutions tout à la fois de l'ordre dominicain et de la pratique de la prédication à une époque où les techniques de communication sont mieux maîtrisées. Tout ceci contribuant à éclairer la «cosmovision " des hommes de la fin du xiII siècle, c'est-àdire leur vision du monde, ce terme venant remplacer celui de «mentalité » auquel l'auteur a renoncé.

2 Hervé Martin épouse les contours et les aspérités du manuel de prédication de Pérégrin d'Opole dont il cite et traduit de très nombreux passages, préférant la pratique de la citation partielle accompagnée d'une explication exhaustive à une traduction linéaire du latin, forcément plus plane. Il s'appuie pour cela sur Perigrini de Opole, Sermones de tempore et de sanctis, édition de Richard Tatarzyński, Institutum Thomisticum, Varsovie, 1997, $650 \mathrm{p}$.

3 Les chapitres I et II, prolégomènes nécessaires à la découverte des horizons mentaux de Pérégrin d'Opole, sont consacrés à la présentation du protagoniste et de son œuvre. Né vers 1260 à Oppeln en Silésie dans une famille citadine, Pérégrin reste tout au long de son existence profondément attaché au monde urbain, préférant les grandes villes germano-polonaises (Opole, Ratibor et Wroclaw) aux campagnes environnantes. Il meurt peu après 1327, laissant une œuvre conséquente composée de 105 sermons « du temps » et 207 sermons « des saints ». Formé à proximité de son lieu de naissance à Opole et Ratibor, il n'a pas fait de grandes études et n'a pas fréquenté les grands foyers universitaires et intellectuels qui font la gloire de l'Europe chrétienne de cette époque, préférant l'inspiration divine aux réflexions dialectiques complexes, ce qui se traduit par le caractère très pragmatique et direct de ses textes, qui ne sont pas obscurcis par les effets de style universitaires. Il est cependant tout à fait capable de prêcher indifféremment en polonais devant la cour ou le peuple, en allemand devant les élites citadines et en latin lorsque ses auditeurs sont des clercs. Sa carrière est brillante : prieur de Ratibor en 1303, puis du grand couvent saint Adalbert de Wroclaw, il devient provincial de son ordre en Pologne de 1305 à 1312. Enfin, son éloquence et sa science théologique lui valent d'être fait inquisiteur en 1317.

4 Son parcours individuel entre en résonance avec son époque : la période de sa vie que Pérégrin a consacrée à la prédication coïncide presque exactement avec ce qui représente pour Hervé Martin un temps d'équilibre, presque d'apogée, de la chrétienté latine. En effet, entre 1280 et 1330, l'Église a pu diffuser par l'entremise des nombreux ordres mendiants les principes et les acquis des Conciles de Latran IV (1215) et de Lyon II (1274), et assurer ainsi un encadrement efficace de tous les fidèles. Même loin de Rome, en Silésie, où pourtant la christianisation de la vie quotidienne et du cycle agraire a été très lente, et où les superstitions et les manifestations de paganisme restent encore nombreuses, l'œuvre de Pérégrin manifeste la réalité de cette imprégnation. Pour que celle-ci soit toujours plus profonde, Pérégrin mène une prédication intense dont ne restent cependant aujourd'hui que 312 sermons. Hervé Martin y voit, plutôt qu'un simple reliquat, un " précipité » (p. 37), au sens chimique du terme (« substance séparée de son solvant et tombée au fond du vase, par l'action d'un réactif que l'on a introduit dans le liquide »), le résultat de choix opérés par Pérégrin lui-même, au moment où, imitant certains de ses prestigieux prédécesseurs comme Berthold de Ratisbonne ( $† 1272$ ) ou Martin le Polonais († 1279), il décide de mettre ses sermons à la disposition de ses confrères prédicateurs. Ce recueil, qui a connu un très grand succès, est conservé dans près de 300 manuscrits à travers toute l'Europe, sans qu'il en existe aucun cependant qui soit de Pérégrin lui-même. Le texte des sermons est 
volontairement très ramassé afin de gagner en efficacité au moment de la recherche de tel ou tel thème et de laisser la possibilité ensuite à l'orateur de broder à partir du canevas proposé. Pérégrin écrit lui-même dans ses sermons que son époque correspond à une acmé de l'activité de prédication, dont le dynamisme est soutenu par la puissance des réseaux dominicains.

Dans le chapitre III, Hervé Martin laisse la parole à Pérégrin dont il cite et commente de larges passages afin de permettre au lecteur de découvrir la tonalité de son discours et les visées de son entreprise. Affleure alors la matière vivante d'un discours rigoureux mais qui ne respecte pas toujours les règles du sermo modernus telles qu'elles ont été définies dans les écoles de théologie au début du XIII ${ }^{\mathrm{e}}$ siècle et qui consiste en un commentaire construit du texte scripturaire ramené à un verset thématique, préférant en proposer une version simplifiée et débarrassée de trop nombreuses citations.

Les chapitres IV, V et VI sont dédiés à la forme et au contenu du discours de Pérégrin. Ils cherchent à en mesurer la portée en montrant de quelle manière le prédicateur, animé par le souci de rendre accessible à tous son message religieux, se fait tour à tour pédagogue ou narrateur. N'étant pas docteur en science sacrée, il évite les subtilités théologiques absconses. Lorsqu'il emprunte aux sources hagiographiques ou à d'autres recueils homilétiques, il condense, le plus souvent, le propos dont il s'inspire. Il délaisse la rhétorique savante au profit d'une transmission plus directe des vérités de la foi, des bases de la morale et des normes de comportement. Il n'hésite pas à avoir recours à la narration afin d'éclairer son propos. Il insère ainsi 138 exempla, dans ses sermons, empruntant à une tradition dont il représente la quatrième génération et à laquelle il n'apporte guère d'innovation, s'appropriant des récits qu'il répète à l'identique, même s'il choisit le plus souvent d'y abolir tous les repères historiques ou géographiques, ne retenant que les aspects les plus universels. Préférant faire peur que faire rire, le prédicateur devenu conteur utilise le discours exemplaire pour effrayer puis rassurer ses auditeurs et les conduire plus aisément vers la Bible. Hervé Martin souligne que les apports anthropologiques des exempla de Pérégrin d'Opole sont moindres que ceux de certains de ses maîtres dans la mesure où l'Église ne cherche pas encore à extirper les superstitions locales de la partie de l'Europe dans laquelle il exerce sa charge. Aussi les vertus cathartiques d'un exemplum qui sert à l'Église à s'approprier puis à éradiquer de vieilles croyances restent ici très secondaires.

7 Les chapitres VII, VIII et IX déterminent les objectifs principaux de ce discours : diffuser la doctrine des grands conciles du XIII ${ }^{\mathrm{e}}$ siècle, donner une nouvelle appréciation de la position sociale des femmes et enfin promouvoir l'ordre dominicain. La conformité des propos de Pérégrin aux canons du Concile de Latran IV (1215) est frappante. Les exemples en sont nombreux, certains sont particulièrement frappants, à l'instar de la présentation explicite de la communion et de la confession comme autant de rouages du « dispositif du salut » que l'Église met à la disposition de ses fidèles. Le christianisme qui apparaît dans ce manuel est humain et fraternel sans être, pour autant, affranchi de toute crainte. Une autre particularité de cette forme de dévotion innovante est le recours presque systématique aux intercesseurs proches des fidèles (les «forces du Bien ») que sont le Christ, la Vierge et les saints. De la forte humanité dont est empreinte ce recueil découle une forme de bienveillance à l'égard des femmes. À l'inverse d'autres prédicateurs, Pérégrin d'Opole «n'a pas enrichi le florilège de la misogynie cléricale ", sans y échapper totalement pour autant. Il insiste sur le rôle que la femme peut jouer dans la société en tant qu'épouse, dont il vante le statut, et en tant 
que mère bien sûr, mais également comme auxiliaire du prédicateur dont elle relaie les sermons jusque dans son propre foyer. Sans être ouvertement féministe, il évoque les saintes femmes avec une dévotion aussi ardente que rare. Son ouvrage a cependant moins vocation à glorifier la féminité qu'à mettre en avant l'ordre auquel il est si fier d'appartenir : celui des frères prêcheurs. Revenant fréquemment sur la personnalité du saint fondateur, il décrit précisément l'esprit missionnaire des origines de son ordre, qui a toute son actualité pour des prédicateurs polonais confrontés à des populations encore peu évangélisées. Et, en même temps, il semble avoir pleinement conscience des transformations de ce même ordre, devenu une corporation de prédicateurs puisque la prédication a acquis le statut de métier qui donne lieu à une rétribution de la part des fidèles, et n'est plus seulement un apostolat ou une mission confiée par Dieu.

8 Le chapitre $\mathrm{X}$, enfin, montre que la conscience aiguë qu'a Pérégrin du monde qui l'entoure le conduit à évoquer dans ses sermons des éléments de la vie quotidienne (rythmes urbains, événements politiques locaux, commerce...) qui sont autant de realia, d'éléments de la vie réelle, propres à capter et à entretenir l'attention de son auditoire. En parfaite adéquation avec son temps, l'orateur dominicain est un guide exceptionnel pour l'historien à travers la Pologne de la fin du XIII ${ }^{\mathrm{e}}$ siècle.

9 Aussi, l'œuvre de Pérégrin est-elle à la fois utile et passionnante. Utile parce qu'elle permet de prendre la mesure de la standardisation du discours religieux de cette époque, passionnante parce qu'elle s'épanouit dans un contexte original. Il convient donc de savoir gré à Hervé Martin d'avoir introduit le texte même de Pérégrin auprès $\mathrm{du}$ public français et d'en rendre intelligibles les moindres détails. Cet ouvrage important vient richement compléter et justifier la démarche actuelle des médiévistes qui consiste à collecter l'intégralité des textes homilétiques, en ne se contentant plus d'en isoler les passages les plus narratifs ou les exempla, longtemps considérés comme les extraits les plus significatifs des sermons, et en considérant le discours des prédicateurs dans son ensemble.

\section{AUTEURS}

\section{ANNE-LAURE LALLOUETTE}

Université Paris-IV Sorbonne. 